\title{
Application of Local Fractional Series Expansion Method to Solve Klein-Gordon Equations on Cantor Sets
}

\author{
Ai-Min Yang, ${ }^{1,2}$ Yu-Zhu Zhang, ${ }^{2,3}$ Carlo Cattani, ${ }^{4}$ Gong-Nan Xie, ${ }^{5}$ \\ Mohammad Mehdi Rashidi, ${ }^{6}$ Yi-Jun Zhou, ${ }^{7}$ and Xiao-Jun Yang ${ }^{8}$ \\ ${ }^{1}$ College of Science, Hebei United University, Tangshan 063009, China \\ ${ }^{2}$ College of Mechanical Engineering, Yanshan University, Qinhuangdao 066004, China \\ ${ }^{3}$ College of Metallurgy and Energy, Hebei United University, Tangshan 063009, China \\ ${ }^{4}$ Department of Mathematics, University of Salerno, Via Ponte don Melillo, Fisciano, 84084 Salerno, Italy \\ ${ }^{5}$ School of Mechanical Engineering, Northwestern Polytechnical University, Xian, Shaanxi 710048, China \\ ${ }^{6}$ Department of Mechanical Engineering, Bu-Ali Sina University, P.O. Box 65175-4161, Hamedan, Iran \\ ${ }^{7}$ Qinggong College, Hebei United University, Tangshan 063009, China \\ ${ }^{8}$ Department of Mathematics and Mechanics, China University of Mining and Technology, Xuzhou, Jiangsu 221008, China \\ Correspondence should be addressed to Yu-Zhu Zhang; zyz@heuu.edu.cn
}

Received 5 January 2014; Revised 8 February 2014; Accepted 8 February 2014; Published 12 March 2014

Academic Editor: Ali H. Bhrawy

Copyright (c) 2014 Ai-Min Yang et al. This is an open access article distributed under the Creative Commons Attribution License, which permits unrestricted use, distribution, and reproduction in any medium, provided the original work is properly cited.

We use the local fractional series expansion method to solve the Klein-Gordon equations on Cantor sets within the local fractional derivatives. The analytical solutions within the nondifferential terms are discussed. The obtained results show the simplicity and efficiency of the present technique with application to the problems of the liner differential equations on Cantor sets.

\section{Introduction}

The Klein-Gordon equation [1] has been applied to mathematical physics such as solid-state physics, nonlinear optics, and quantum field theory. Some of the analytical methods for solving the Klein-Gordon equation include the variational iteration method [2], the tanh and the sine-cosine methods [3], the decomposition method [4], the differential transform method [5], and the homotopy-perturbation method [6].

Recently, the solutions for the fractional Klein-Gordon equation with the Caputo fractional derivative were considered in [7-9]. Golmankhaneh et al. used the homotopyperturbation method to obtain solution for the fractional Klein-Gordon equation [7]. Kurulay [8] pointed out the solution for the fractional Klein-Gordon equation by using the homotopy analysis method. Gepreel and Mohamed [9] presented the solution for nonlinear space-time fractional Klein-Gordon equation by the homotopy analysis method.

When some domains cannot be described by smooth functions, both the classical approach and the fractional approach based on Riemann-Liouville (or Caputo) derivatives are unacceptable. In such cases, the local fractional calculus is an efficient technique for modeling these physical problems [10-23]. Using the fractional complex transform method [20], one transforms the classical Klein-Gordon equation into the Klein-Gordon equation on Cantor sets in the following form:

$$
\frac{\partial^{2 \alpha} u(x, t)}{\partial t^{2 \alpha}}-\frac{\partial^{2 \alpha} u(x, t)}{\partial x^{2 \alpha}}=F(u(x)),
$$

subject to the initial value conditions:

$$
\begin{gathered}
u(x, 0)=f(x), \\
\frac{\partial^{\alpha}}{\partial u^{\alpha}} u(x, 0)=g(x),
\end{gathered}
$$


where the operator is the local fractional derivative operator, which is defined by [16-23]

$$
f^{(\alpha)}\left(x_{0}\right)=\left.\frac{d^{\alpha} f(x)}{d x^{\alpha}}\right|_{x=x_{0}}=\lim _{x \rightarrow x_{0}} \frac{\Delta^{\alpha}\left(f(x)-f\left(x_{0}\right)\right)}{\left(x-x_{0}\right)^{\alpha}}
$$

with $\Delta^{\alpha}\left(f(x)-f\left(x_{0}\right)\right) \cong \Gamma(1+\alpha) \Delta\left(f(x)-f\left(x_{0}\right)\right)$ and $u(x, t)$ and $g(x)$ are the local fractional continuous functions and $F(u(x))$ are the mixed terms of nonlinear and liner functions.

In view of (1)-(2), the linear Klein-Gordon equation on Cantor sets:

$$
\frac{\partial^{2 \alpha} u(x, t)}{\partial t^{2 \alpha}}-\frac{\partial^{2 \alpha} u(x, t)}{\partial x^{2 \alpha}}=u(x, t), \quad x>0, t>0,
$$

subject to the initial value conditions:

$$
\begin{gathered}
u(x, 0)=f(x), \\
\frac{\partial^{\alpha}}{\partial u^{\alpha}} u(x, 0)=g(x),
\end{gathered}
$$

is under consideration, where $f(x)$ and $g(x)$ are local fractional continuous functions.

On the other hand, the local fractional series expansion method was applied to solve the wave and diffusion equations on Cantor sets [21], the local fractional Schrödinger equation in the one-dimensional Cantorian system [22], and the local fractional Helmholtz equation [23]. In this paper, our aim is to investigate a new application of this technology to solve the linear Klein-Gordon equations on Cantor sets. The paper is organized as follows. In Section 2, the idea of local fractional series expansion method is given. In Section 3, the solutions for linear Klein-Gordon equations on Cantor sets are presented. Finally, Section 4 is the conclusions.

\section{The Local Fractional Series Expansion Method}

In order to illustrate the idea of the local fractional series expansion method [21-23], we consider the local fractional differential operator equation in the following form:

$$
u_{2 t}^{2 \alpha}=L_{\alpha} u
$$

where $L_{\alpha}$ is the linear local fractional operator and $\phi$ is a local fractional continuous function.

From (6), the multiterm separated functions with respect to $x, t$ read as

$$
u(x, t)=\sum_{i=0}^{\infty} \phi_{i}(t) \psi_{i}(x)
$$

where $\phi_{i}(t)$ and $\psi_{i}(x)$ are the local fractional continuous functions.

From (7), we have

$$
\phi_{i}(t)=\frac{t^{i \alpha}}{\Gamma(1+i \alpha)},
$$

so that

$$
u(x, t)=\sum_{i=0}^{\infty} \frac{t^{i \alpha}}{\Gamma(1+i \alpha)} \psi_{i}(x)
$$

In view of (9), we obtain

$$
\begin{gathered}
u_{t}^{2 \alpha}=\sum_{i=0}^{\infty} \frac{1}{\Gamma(1+i \alpha)} t^{i \alpha} \psi_{i+2}(x), \\
L_{\alpha} u=L_{\alpha}\left[\sum_{i=0}^{\infty} \frac{t^{i \alpha}}{\Gamma(1+i \alpha)} \psi_{i}(x)\right]=\sum_{i=0}^{\infty} \frac{t^{i \alpha}}{\Gamma(1+i \alpha)}\left(L_{\alpha} \psi_{i}\right)(x) .
\end{gathered}
$$

Making use of (10), we have

$$
\sum_{i=0}^{\infty} \frac{1}{\Gamma(1+i \alpha)} t^{i \alpha} \psi_{i+2}(x)=\sum_{i=0}^{\infty} \frac{t^{i \alpha}}{\Gamma(1+i \alpha)}\left(L_{\alpha} \psi_{i}\right)(x),
$$

so that

$$
\psi_{i+2}(x)=\left(L_{\alpha} \psi_{i}\right)(x) .
$$

Hence, from (12) we get

$$
u(x, t)=\sum_{i=0}^{\infty} u_{i}(x, t)=\sum_{i=0}^{\infty} \frac{t^{i \alpha}}{\Gamma(1+i \alpha)} \psi_{i}(x)
$$

We now rewrite (4) in the local fractional operator form as follows:

$$
u_{2 t}^{2 \alpha}=L_{\alpha} u
$$

subject to the initial value conditions:

$$
\begin{gathered}
u(x, 0)=f(x), \\
\frac{\partial^{\alpha}}{\partial u^{\alpha}} u(x, 0)=g(x),
\end{gathered}
$$

where the linear local fractional operator is defined as follows:

$$
L_{\alpha}=\frac{\partial^{2 \alpha}}{\partial x^{2 \alpha}}+I
$$

Hence, (16) is a special case of (6) and it is used with the linear Klein-Gordon equations on Cantor sets in next section.

\section{Analytical Solutions for Linear Klein-Gordon Equations on Cantor Sets}

In this section, we present the nondifferentiable solutions for linear Klein-Gordon equations on Cantor sets.

Example 1. Let us consider the Klein-Gordon equations on Cantor sets in the following form:

$$
u_{2 t}^{2 \alpha}=L_{\alpha} u
$$


subject to the initial value conditions:

$$
\begin{gathered}
u(x, 0)=0, \\
\frac{\partial^{\alpha}}{\partial u^{\alpha}} u(x, 0)=\frac{x^{2 \alpha}}{\Gamma(1+2 \alpha)} .
\end{gathered}
$$

From (12) and (18), we can structure the following iterative formulas:

$$
\begin{gathered}
\psi_{i+2}(x)=\left(\frac{\partial^{2 \alpha} \psi_{i}}{\partial x^{2 \alpha}}+\psi_{i}\right)(x), \\
\psi_{0}(x)=0, \\
\psi_{i+2}(x)=\left(\frac{\partial^{2 \alpha} \psi_{i}}{\partial x^{2 \alpha}}+\psi_{i}\right)(x), \\
\psi_{1}(x)=\frac{x^{2 \alpha}}{\Gamma(1+2 \alpha)} .
\end{gathered}
$$

Hence, we can calculate

$$
\begin{aligned}
& \psi_{0}(x)=0, \\
& \psi_{1}(x)=\frac{x^{2 \alpha}}{\Gamma(1+2 \alpha)}, \\
& \psi_{2}(x)=0, \\
& \psi_{3}(x)=1+\frac{x^{2 \alpha}}{\Gamma(1+2 \alpha)}, \\
& \psi_{4}(x)=0, \\
& \psi_{5}(x)=1+\frac{x^{2 \alpha}}{\Gamma(1+2 \alpha)},
\end{aligned}
$$

and so on.

Therefore, we have

$$
\begin{aligned}
u(x, t)= & \frac{x^{2 \alpha}}{\Gamma(1+2 \alpha)} \sum_{i=1}^{\infty} \frac{t^{(2 i-1) \alpha}}{\Gamma(1+(2 i-1) \alpha)} \\
& +\sum_{i=1}^{\infty} \frac{t^{(1+2 i) \alpha}}{\Gamma(1+(1+2 i) \alpha)},
\end{aligned}
$$

and the corresponding graph is illustrated in Figure 1.

Example 2. We consider the following Klein-Gordon equations on Cantor sets:

$$
u_{2 t}^{2 \alpha}=L_{\alpha} u
$$

subject to the initial value conditions:

$$
\begin{gathered}
u(x, 0)=\frac{x^{\alpha}}{\Gamma(1+\alpha)}, \\
\frac{\partial^{\alpha}}{\partial u^{\alpha}} u(x, 0)=0 .
\end{gathered}
$$

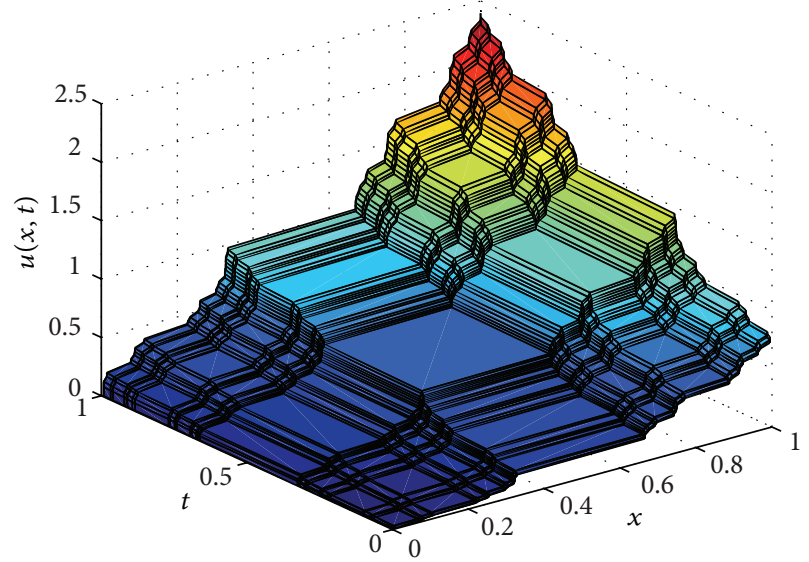

Figure 1: The plot of $u(x, t)$ for the parameter $\alpha=\ln 2 / \ln 3$.

From (12) and (23), we get the following iterative formulas:

$$
\begin{gathered}
\psi_{i+2}(x)=\left(\frac{\partial^{2 \alpha} \psi_{i}}{\partial x^{2 \alpha}}+\psi_{i}\right)(x), \\
\psi_{0}(x)=\frac{x^{\alpha}}{\Gamma(1+\alpha)}, \\
\psi_{i+2}(x)=\left(\frac{\partial^{2 \alpha} \psi_{i}}{\partial x^{2 \alpha}}+\psi_{i}\right)(x), \\
\psi_{1}(x)=0 .
\end{gathered}
$$

Hence, we get

$$
\begin{aligned}
& \psi_{0}(x)=\frac{x^{\alpha}}{\Gamma(1+\alpha)}, \\
& \psi_{1}(x)=0 \\
& \psi_{2}(x)=\frac{x^{\alpha}}{\Gamma(1+\alpha)}, \\
& \psi_{3}(x)=0, \\
& \psi_{4}(x)=\frac{x^{\alpha}}{\Gamma(1+\alpha)}, \\
& \psi_{5}(x)=0
\end{aligned}
$$

and so on.

Hereby, we obtain the solution of (22):

$$
u(x, t)=\frac{x^{\alpha}}{\Gamma(1+\alpha)} \sum_{i=0}^{\infty} \frac{t^{2 i \alpha}}{\Gamma(1+2 i \alpha)},
$$

and the corresponding graph is depicted in Figure 2.

Example 3. We present the following Klein-Gordon equations on Cantor sets:

$$
u_{2 t}^{2 \alpha}=L_{\alpha} u
$$




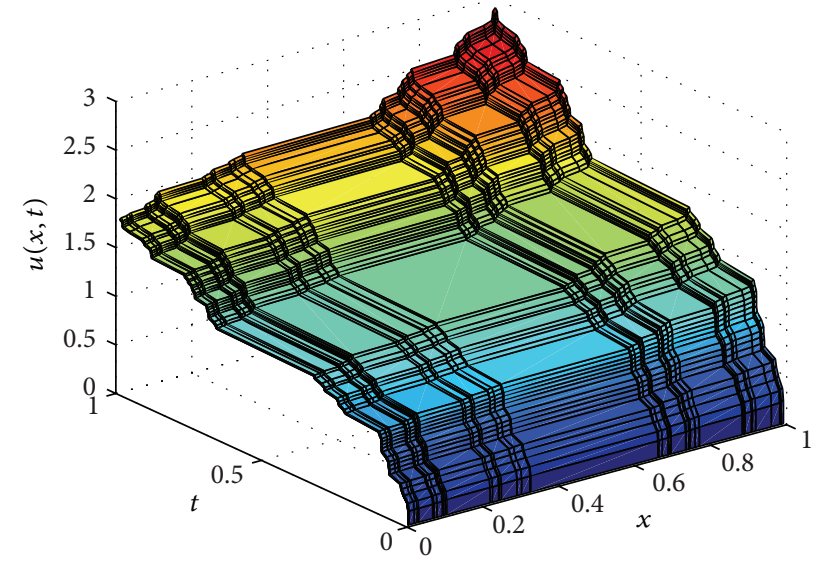

Figure 2: The plot of $u(x, t)$ for the parameter $\alpha=\ln 2 / \ln 3$.

subject to the initial value conditions:

$$
\begin{gathered}
u(x, 0)=\frac{x^{2 \alpha}}{\Gamma(1+2 \alpha)}, \\
\frac{\partial^{\alpha}}{\partial u^{\alpha}} u(x, 0)=\frac{x^{2 \alpha}}{\Gamma(1+2 \alpha)} .
\end{gathered}
$$

From (12) and (27)-(28), we get the following iterative formulas:

$$
\begin{gathered}
\psi_{i+2}(x)=\left(\frac{\partial^{2 \alpha} \psi_{i}}{\partial x^{2 \alpha}}+\psi_{i}\right)(x), \\
\psi_{0}(x)=\frac{x^{2 \alpha}}{\Gamma(1+\alpha)}, \\
\psi_{i+2}(x)=\left(\frac{\partial^{2 \alpha} \psi_{i}}{\partial x^{2 \alpha}}+\psi_{i}\right)(x), \\
\psi_{1}(x)=\frac{x^{2 \alpha}}{\Gamma(1+2 \alpha)} .
\end{gathered}
$$

From (29) we obtain

$$
\begin{aligned}
& \psi_{0}(x)=\frac{x^{2 \alpha}}{\Gamma(1+2 \alpha)}, \\
& \psi_{1}(x)=\frac{x^{2 \alpha}}{\Gamma(1+2 \alpha)}, \\
& \psi_{2}(x)=1+\frac{x^{2 \alpha}}{\Gamma(1+2 \alpha)}, \\
& \psi_{3}(x)=1+\frac{x^{2 \alpha}}{\Gamma(1+2 \alpha)}, \\
& \psi_{4}(x)=1+\frac{x^{2 \alpha}}{\Gamma(1+2 \alpha)}, \\
& \psi_{5}(x)=1+\frac{x^{2 \alpha}}{\Gamma(1+2 \alpha)},
\end{aligned}
$$

and so on.
Therefore, we obtain the exact solution of (27)

$$
u(x, t)=\frac{x^{\alpha}}{\Gamma(1+\alpha)} E_{\alpha}\left(t^{\alpha}\right)+E_{\alpha}\left(t^{\alpha}\right)-\frac{t^{\alpha}}{\Gamma(1+\alpha)}-1,
$$

and its graph is shown in Figure 3.

Example 4. The Klein-Gordon equation on Cantor sets is presented as

$$
u_{2 t}^{2 \alpha}=L_{\alpha} u,
$$

and the initial value conditions are written as

$$
\begin{gathered}
u(x, 0)=\frac{x^{\alpha}}{\Gamma(1+\alpha)}, \\
\frac{\partial^{\alpha}}{\partial u^{\alpha}} u(x, 0)=\frac{x^{\alpha}}{\Gamma(1+\alpha)} .
\end{gathered}
$$

From (12) and (27)-(28), the following iterative formulas are as follows:

$$
\begin{gathered}
\psi_{i+2}(x)=\left(\frac{\partial^{2 \alpha} \psi_{i}}{\partial x^{2 \alpha}}+\psi_{i}\right)(x), \\
\psi_{0}(x)=\frac{x^{\alpha}}{\Gamma(1+\alpha)}, \\
\psi_{i+2}(x)=\left(\frac{\partial^{2 \alpha} \psi_{i}}{\partial x^{2 \alpha}+\psi_{i}}\right)(x), \\
\psi_{1}(x)=\frac{x^{\alpha}}{\Gamma(1+\alpha)} .
\end{gathered}
$$

From (29), we give

$$
\begin{aligned}
& \psi_{0}(x)=\frac{x^{\alpha}}{\Gamma(1+\alpha)}, \\
& \psi_{1}(x)=\frac{x^{\alpha}}{\Gamma(1+\alpha)}, \\
& \psi_{2}(x)=\frac{x^{\alpha}}{\Gamma(1+\alpha)}, \\
& \psi_{3}(x)=\frac{x^{\alpha}}{\Gamma(1+\alpha)}, \\
& \psi_{4}(x)=\frac{x^{\alpha}}{\Gamma(1+\alpha)}, \\
& \psi_{5}(x)=\frac{x^{\alpha}}{\Gamma(1+\alpha)},
\end{aligned}
$$

and so on.

Therefore, we give the exact solution of (32):

$$
u(x, t)=\frac{x^{\alpha}}{\Gamma(1+\alpha)} E_{\alpha}\left(t^{\alpha}\right),
$$

and its graph is shown in Figure 4. 


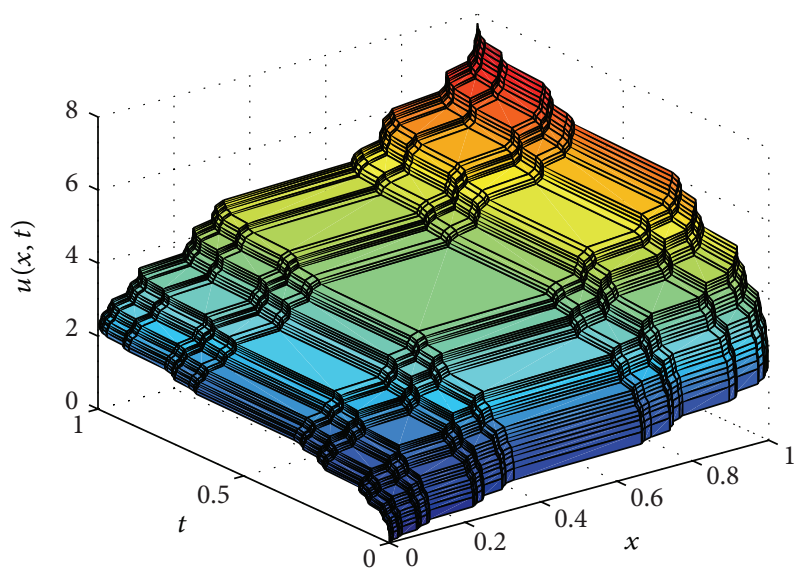

Figure 3: The plot of $u(x, t)$ for the parameter $\alpha=\ln 2 / \ln 3$.

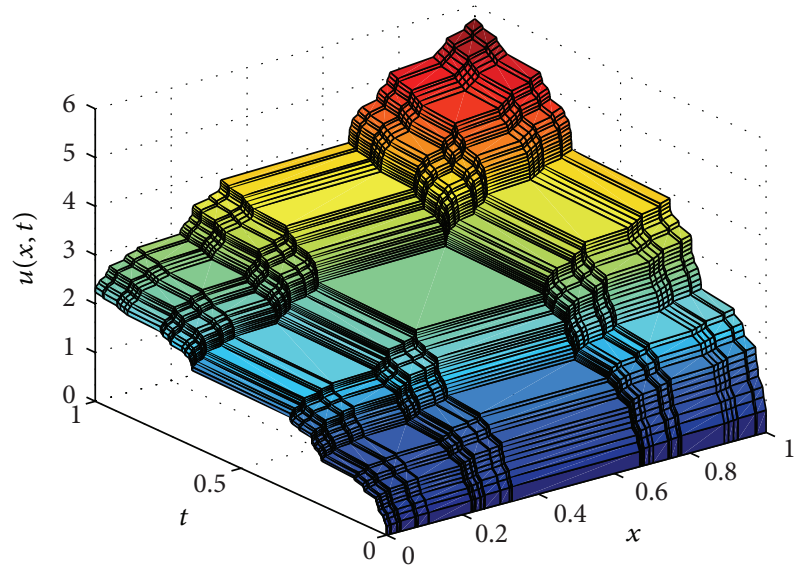

Figure 4: The plot of $u(x, t)$ for the parameter $\alpha=\ln 2 / \ln 3$.

\section{Conclusions}

In this work the Klein-Gordon equations on Cantor sets within the local fractional differential operator had been analyzed using the local fractional series expansion method. The nondifferentiable solutions for local fractional KleinGordon equations were obtained. The present method is a powerful mathematical tool for solving the local fractional linear differential equations.

\section{Conflict of Interests}

The authors declare that they have no conflict of interests regarding the publication of this paper.

\section{Acknowledgments}

This work was supported by National Scientific and Technological Support Projects (no. 2012BAE09B00), the National Natural Science Foundation of China (no. 51274270), and the National Natural Science Foundation of Hebei Province (no. E2013209215).

\section{References}

[1] A.-M. Wazwaz, "Compactons, solitons and periodic solutions for some forms of nonlinear Klein-Gordon equations," Chaos, Solitons \& Fractals, vol. 28, no. 4, pp. 1005-1013, 2006.

[2] E. Yusufoğlu, "The variational iteration method for studying the Klein-Gordon equation," Applied Mathematics Letters, vol. 21, no. 7, pp. 669-674, 2008.

[3] A.-M. Wazwaz, "The tanh and the sine-cosine methods for compact and noncompact solutions of the nonlinear KleinGordon equation," Applied Mathematics and Computation, vol. 167, no. 2, pp. 1179-1195, 2005.

[4] S. M. El-Sayed, "The decomposition method for studying the Klein-Gordon equation," Chaos, Solitons \& Fractals, vol. 18, no. 5, pp. 1025-1030, 2003.

[5] A. S. V. Ravi Kanth and K. Aruna, "Differential transform method for solving the linear and nonlinear Klein-Gordon equation," Computer Physics Communications, vol. 180, no. 5, pp. 708-711, 2009.

[6] M. S. H. Chowdhury and I. Hashim, "Application of homotopyperturbation method to Klein-Gordon and sine-Gordon equations," Chaos, Solitons \& Fractals, vol. 39, no. 4, pp. 1928-1935, 2009.

[7] A. K. Golmankhaneh, A. K. Golmankhaneh, and D. Baleanu, "On nonlinear fractional KleinGordon equation," Signal Processing, vol. 91, no. 3, pp. 446-451, 2011.

[8] M. Kurulay, "Solving the fractional nonlinear Klein-Gordon equation by means of the homotopy analysis method," Advances in Difference Equations, vol. 2012, no. 1, article 187, pp. 1-8, 2012.

[9] K. A. Gepreel and M. S. Mohamed, "Analytical approximate solution for nonlinear space-time fractional Klein-Gordon equation," Chinese Physics B, vol. 22, no. 1, Article ID 010201, 2013.

[10] K. M. Kolwankar and A. D. Gangal, "Local fractional FokkerPlanck equation,” Physical Review Letters, vol. 80, no. 2, pp. 214217, 1998.

[11] A. Carpinteri, B. Chiaia, and P. Cornetti, "Static-kinematic duality and the principle of virtual work in the mechanics of fractal media," Computer Methods in Applied Mechanics and Engineering, vol. 191, no. 1-2, pp. 3-19, 2001.

[12] A. K. Golmankhaneh and D. Baleanu, "On a new measure on fractals," Journal of Inequalities and Applications, vol. 2013, no. 1, article 522, 2013.

[13] A. K. Golmankhaneh, A. K. Golmankhaneh, and D. Baleanu, "Lagrangian and Hamiltonian mechanics on fractals subset of real-line," International Journal of Theoretical Physics, vol. 52, no. 11, pp. 4210-4217, 2013.

[14] K. G. Alireza, V. Fazlollahi, and D. Baleanu, "Newtonian mechanics on fractals subset of real-line," Romania Reports in Physics, vol. 65, pp. 84-93, 2013.

[15] A. S. Balankin, "Stresses and strains in a deformable fractal medium and in its fractal continuum model," Physics Letters A, vol. 377, no. 38, pp. 2535-2541, 2013.

[16] X. J. Yang, Advanced Local Fractional Calculus and Its Applications, World Science Publisher, New York, NY, USA, 2012.

[17] A. M. Yang, Y. Z. Zhang, and Y. Long, "The Yang-Fourier transforms to heat-conduction in a semi-infinite fractal bar," Thermal Science, vol. 17, no. 3, pp. 707-713, 2013.

[18] S. Q. Wang, Y. J. Yang, and H. K. Jassim, "Local fractional function decomposition method for solving inhomogeneous wave equations with local fractional derivative," Abstract and Applied Analysis, vol. 2014, Article ID 176395, 7 pages, 2014. 
[19] J.-H. He, "Exp-function method for fractional differential equations," International Journal of Nonlinear Sciences and Numerical Simulation, vol. 14, no. 6, pp. 363-366, 2013.

[20] X.-J. Yang, D. Baleanu, and J.-H. He, “Transport equations in fractal porous media within fractional complex transform method," Proceedings of the Romanian Academy A, vol. 14, no. 4, pp. 287-292, 2013.

[21] A.-M. Yang, X.-J. Yang, and Z.-B. Li, "Local fractional series expansion method for solving wave and diffusion equations on Cantor sets," Abstract and Applied Analysis, vol. 2013, Article ID 351057, 5 pages, 2013.

[22] Y. Zhao, D.-F. Cheng, and X.-J. Yang, "Approximation solutions for local fractional Schrödinger equation in the onedimensional Cantorian system," Advances in Mathematical Physics, vol. 2013, Article ID 291386, 5 pages, 2013.

[23] A. M. Yang, Z. S. Chen, H. M. Srivastava, and X. -J. Yang, "Application of the local fractional series expansion method and the variational iteration method to the Helmholtz equation involving local fractional derivative operators," Abstract and Applied Analysis, vol. 2013, Article ID 259125, 6 pages, 2013. 


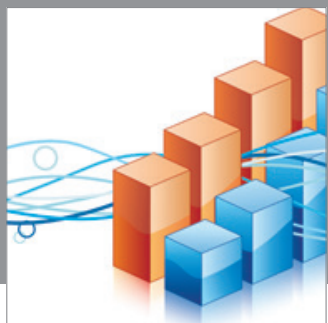

Advances in

Operations Research

mansans

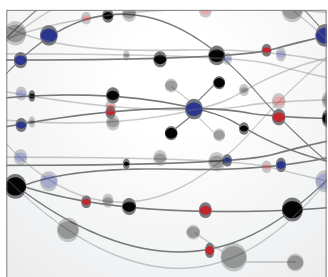

The Scientific World Journal
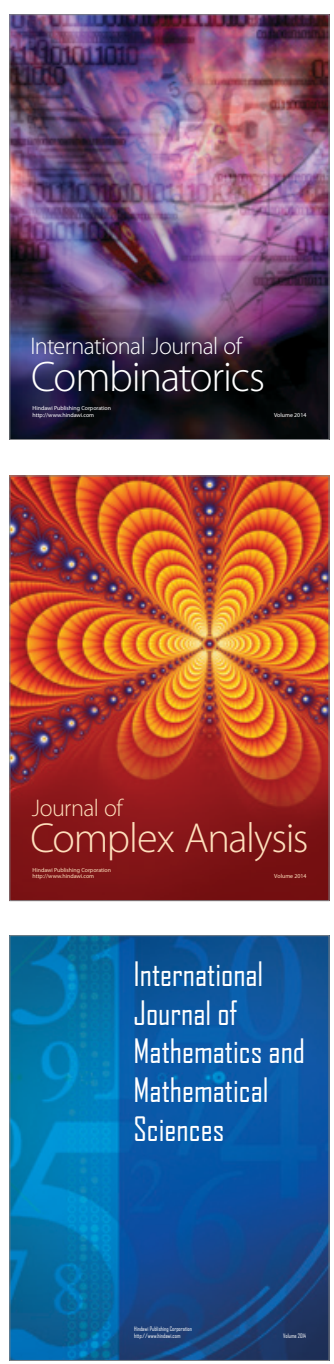
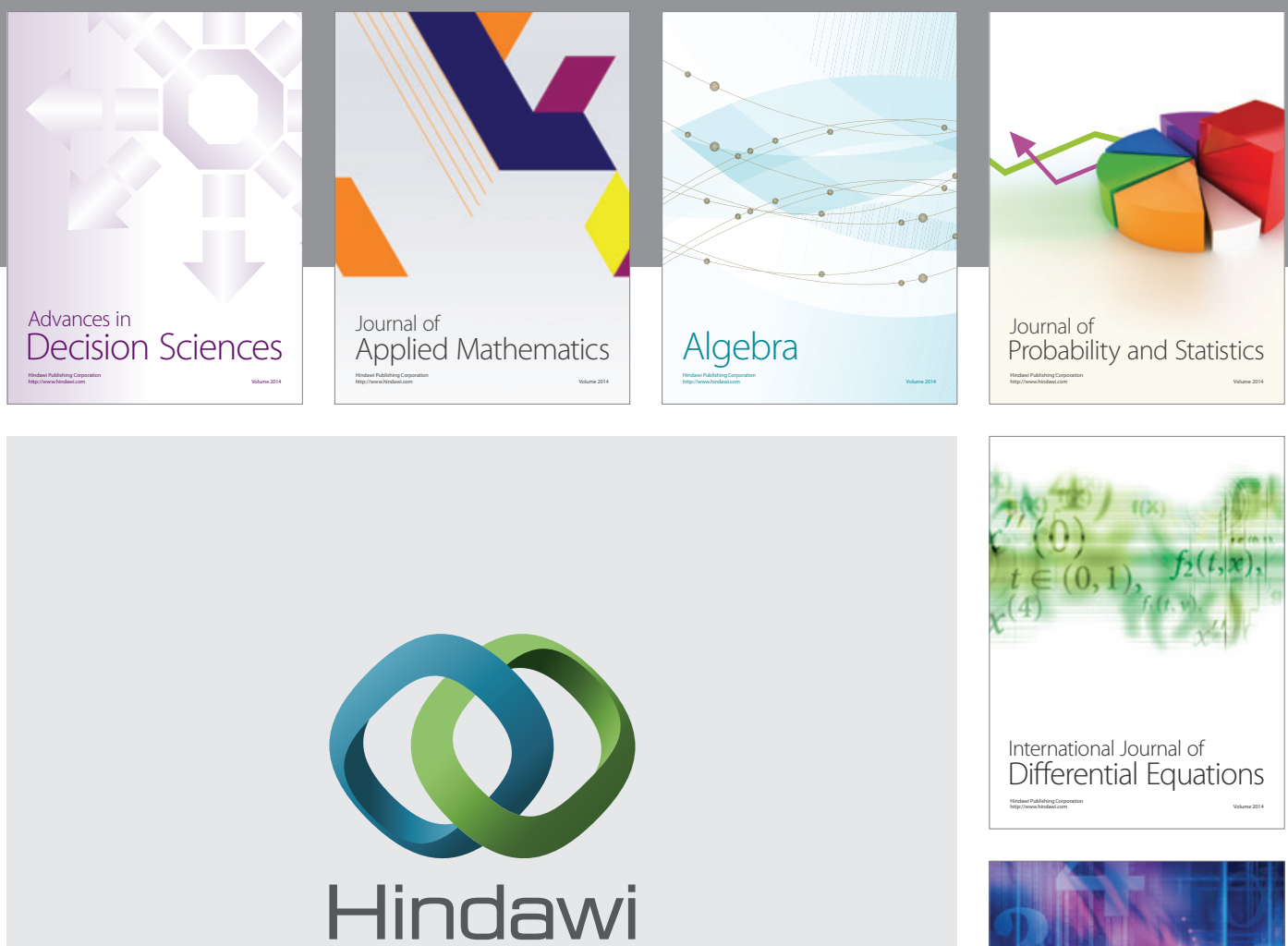

Submit your manuscripts at http://www.hindawi.com
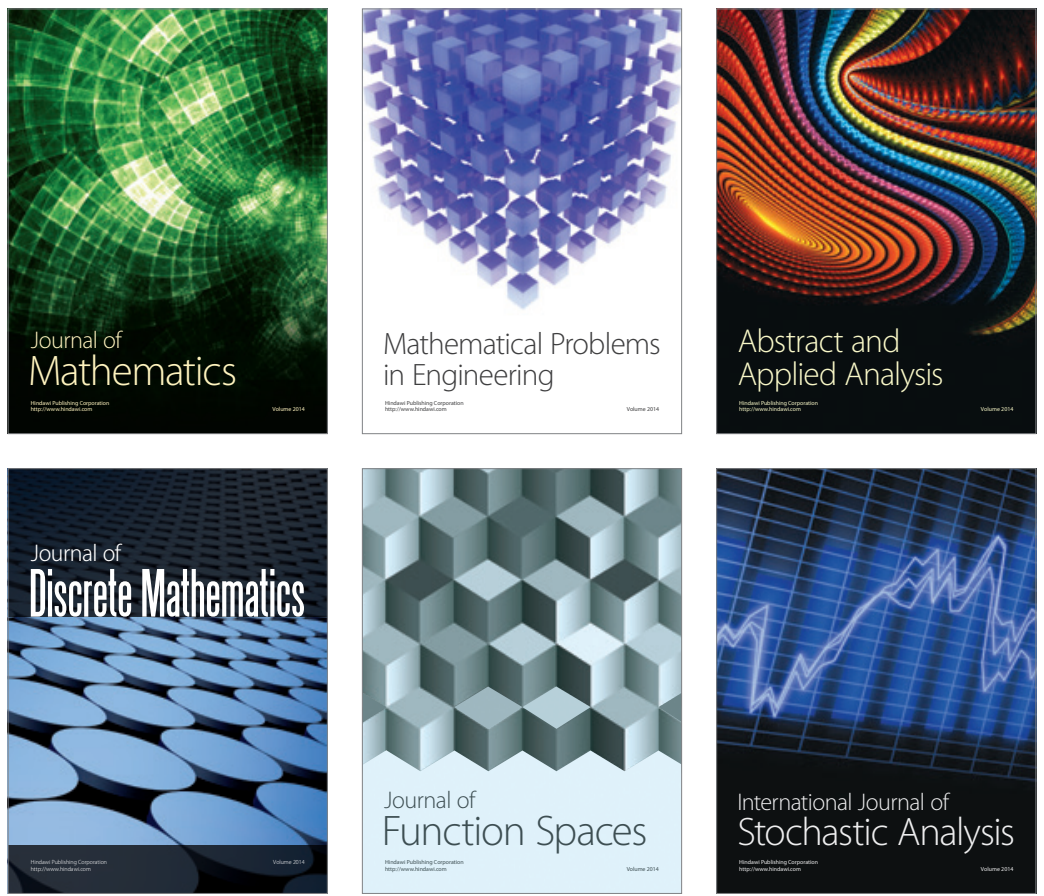

Journal of

Function Spaces

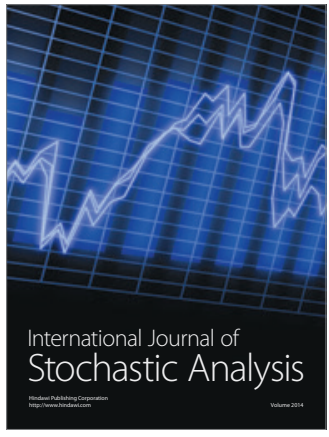

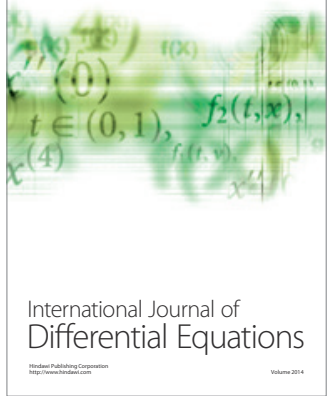
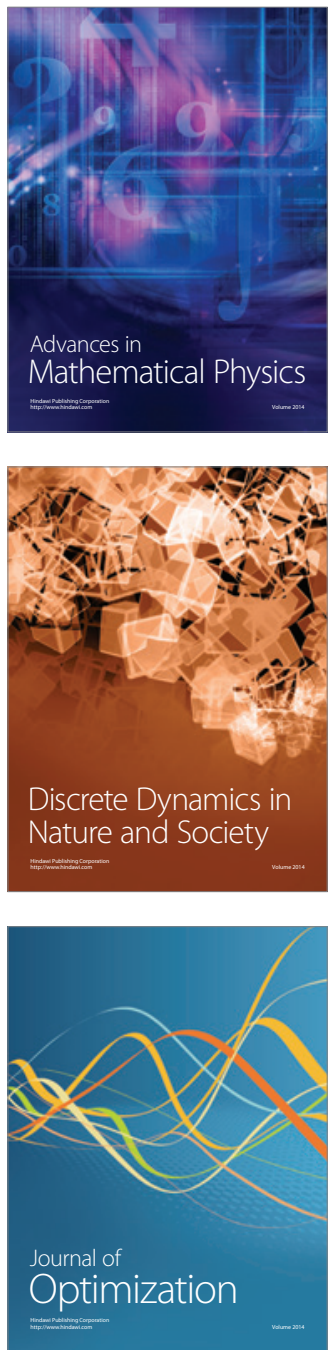